\title{
EVALUACIÓN DE DIETAS ISOENERGÉTICAS CON VARIOS NIVELES DE PROTEÍNA Y DE HARINA DE PESCADO EN ALEVINOS DE BOCACHICO (Prochilodus magdalenae)
}

\section{EVALUATION OF ISOENERGENETIC DIETS WITH VARIOUS LEVELS OF PROTEIN AND FISH MEAL IN BOCACHICO FRIES (Prochilodus magdalenae)}

\begin{abstract}
René Adolfo González Uribe ${ }^{1}$, Gustavo Álvaro Wills Franco²
${ }^{1}$ Zootecnista M.Sc. e-mail: ragonzalezur@unal.edu.co. Asesorías particulares, ${ }^{2}$ Zootecnista M.Sc, Ph.D. e-mail: gawillsf@ unal.edu.co. Docente Facultad de Medicina Veterinaria y de Zootecnia, Universidad Nacional de Colombia. Bogotá, D.C.
\end{abstract}

Rev. U.D.C.A Act. \& Div. Cient. 12 (2): 69-77, 2009

\section{RESUMEN}

Para medir el efecto de tres niveles de proteína cruda (PC) $(24,28$ y 32\%), con dos niveles de de harina de pescado (HP) (7,5 y 0\%), en dietas isoenergéticas (3100 Kcal/kg Energía Digestible Calculada EDC), con cantidades similares de cinco aminoácidos esenciales (lisina, metionina + cistina, triptófano y treonina), en el desempeño de los alevinos de bocachico (Prochilodus magdalenae), se planteó un experimento que tuvo una duración de 32 días. Se utilizó un diseño completamente al azar, con un arreglo factorial de $3 * 2$ (tres niveles de proteína y dos niveles de HP), debido a una diferencia de pesos y de longitudes estándar iníciales; se realizó un ANACOVA para valorar el efecto de las dietas en el peso final, ganancia de peso expresado como porcentaje, tasa de crecimiento específico y ganancia en longitud estándar. Finalmente, se concluyó que no hubo diferencias significativas $(P>0,05)$, entre las diferentes dietas en los parámetros evaluados, por lo tanto, se puede afirmar, que los alevinos de bocachico toleran bien las dietas carentes de la harina de pescado, siempre y cuando cuenten con un buen balance de aminoácidos; además, pueden mostrar un buen desempeño con dietas con una PC del $24 \%$.
Palabras clave: Nutrición alevinos, bocachico, harina de pescado, proteína, aminoácidos esenciales.

\section{SUMMARY}

To measure the effect of three protein levels (24, 28 and $32 \%$ ) with and without the incorporation of fishmeal HP (7.5 and 0\%), isoenergy diets $(3100 \mathrm{Kcal} / \mathrm{kg})$, with similar amounts of five essential amino acids (lysine, methionine + cystine, threonine and tryptophan) in the performance of bocachico (Prochilodus magdalenae) fries, in an experiment of 32 days was performed. For this purpose a completely randomized design with a factorial arrangement $3 * 2$ (three levels of protein and two levels of HP) due to a difference in initial weights and standard lengths was employed. An ANACOVA, to assess the effect of the six diets in the final weight, weight gain expressed as percentage, specific growth rate and gain in standard length was executed. Finally, it was concluded that no significant difference $(P>0.05)$ among the different diets on these parameters was detected, so it can be stated that bocachico fries well tolerated diets devoid of fish meal as long as they have with a good balance of amino acids; furthermore, they can show also a good performance with diets with protein content of $24 \%$. 
Key words: Fry nutrition, bocachico, fishmeal, protein, essential amino acids.

\section{INTRODUCCIÓN}

El bocachico, Prochilodus magdalenae, es un pez que pertenece a la súper familia Characoidae, al género XLIII Prochilodus (Agassiz, 1829). Su boca, es muy pequeña y con pocos dientes alrededor de los labios (Miles, 1974). En la actualidad el bocachico es una de las especies con mayor grado de vulnerabilidad en la cuenca del río Magdalena, por el exceso de la pesca comercial y de su consumo. Su situación es supremamente alarmante, pues se estima que el volumen de captura ha descendido en un $90 \%$ en los últimos 25 años, por lo cual, es evidente que la sobre pesca es el principal factor que ha incidido en la drástica disminución de sus volúmenes y consecuente reducción continua en las tallas medias de captura (Mojica E Álvarez-León, 2002). Según Barreto E Mosquera (2001), el bocachico esta dentro de las cuatro especies más cultivadas en el país, pero uno de los grandes inconvenientes, con los cuales se enfrentan los acuicultores, es la ausencia de dietas diseñadas para esta especie, unido a los altos costos que representa este rubro, debido a la inclusión de la harina de pescado. Según la FAO (2006), la producción mundial de harina de pescado es de seis a siete millones de toneladas, donde la mayor parte de ésta, se usa para la acuicultura, la alimentación del ganado y en el sector avícola. Mientras esta demanda siga creciendo, se intensificará la competición con las especies productivas terrestres por unos recursos limitados, con consecuencias, tanto en los precios como en la disponibilidad.

La harina de pescado (HP) elaborada con materia prima de excelente calidad es la principal fuente de proteína para la preparación de alimentos destinados al consumo de los peces. Es rica en energía y en minerales, de alta digestibilidad y muy palatable para la mayoría de peces; contiene entre 60 y $80 \%$ de proteína cruda (PC), de la cual el 80 al $95 \%$ es digestible para los peces; se caracteriza por su alto contenido de lisina y metionina, los dos aminoácidos más limitantes en los alimentos de origen vegetal (Lovell, 1989; Kikuchi E Furuta, 2009; Civera et al. 2006; Li et al. 2006). Sin embargo, los elevados costos de la harina de pescado, ocasionados por la alta demanda del producto, han obligado a los productores a buscar nuevas alternativas de alimentación con dietas, en las que se incluya muy poca cantidad de ésta (Li et al. 2006; Gaber, 2006) o en casos extremos, a no utilizarla.

Otro problema que se registra es el alto nivel de proteína que se maneja en las dietas de la mayoría de las especies en los sistemas acuícolas (Abimorad et al. 2009), lo que ocasiona también un mayor costo y una contaminación de los cuerpos de agua por los desechos nitrogenados, como lo afirman Salhi et al. (2004) y Abdel-Tawwab E Ahmad (2009). Es así que Zarate E Lovell (1997) consideran que el porcentaje de proteína de la dieta se puede disminuir siempre y cuando se incremente el contenido de los aminoácidos esenciales en el alimento suministrado. Twibell et al. (2003) demostraron que los peces, como otros animales, no tienen un verdadero requerimiento de proteína, pero tienen un gusto por las mezclas bien balanceadas de aminoácidos esenciales y no esenciales. Yamamoto et al. (2000) aclararon que los peces muestran preferencias por las dietas con un buen balance de aminoácidos sobre aquellas con desbalance de éstos. En los últimos años, los investigadores se han centrado en determinar el perfil de aminoácidos adecuado para algunas especies de peces, de tal manera que permita maximizar el crecimiento y la utilización de la proteína (Twibell et al. 2003; Furuya et al. 2004). La inclusión de aminoácidos sintéticos en las dietas para peces ha reportado retardo en el crecimiento (Watanabe et al. 2001) y disminución en la utilización del nitrógeno (Peres \& Oliva-Teles, 2005).

El objeto de este estudio fue la evaluación del desempeño de los alevinos de bocachico (P. magdalenae), sometidos a seis dietas isoenergéticas (igual cantidad de energía), bajo el efecto de tres niveles de PC (24\%, $28 \%$ y $32 \%)$, con dos niveles de HP (7,5 y $0 \%)$ y además, manteniéndolas iguales en los aminoácidos lisina, metionina + cistina, treonina y triptófano, mediante el uso de aminoácidos sintéticos.

\section{MATERIALES Y MÉTODOS}

Animales experimentales: Se dispuso de 72 alevinos con pesos y longitudes heterogéneas, que fueron recibidos en un acuario, con una capacidad de 300L, donde permanecieron por una semana; luego de lo cual fueron repartidos en veinticuatro acuarios de experimentación del Laboratorio de Ictiología de la Facultad de Medicina Veterinaria y de Zootecnia de la Universidad Nacional de Colombia, sede Bogotá. A todos los animales se les determinó, el peso, la longitud total y la longitud estándar 
y es así como se lograron establecer dos grupos. Después de ser ubicados en los acuarios correspondientes, los peces fueron sometidos a un proceso de adaptación, que duró doce días. Se depositaron tres alevinos por acuario, con peso de 4,16 $\pm 0,04 \mathrm{~g}$ en doce acuarios $\mathrm{y}$, en los otros doce, el peso promedio fue de 1,41 \pm $0,02 \mathrm{~g}$. Cada tratamiento tuvo cuatro réplicas.

Preparación de las dietas: Se elaboraron seis dietas con tres niveles de PC 24, 28 y 32\%, con dos niveles de HP (7,5 y 0\%), pero manteniéndolas isoenergéticas, similares en energía, (3100 Kcal/kg.E.D.C.) y contenidos aproximados de los aminoácidos lisina, metionina + cistina, treonina y triptófano (para lo cual, se utilizaron aminoácidos sintéticos), según lo recomendado por González \& Wills (2005).

En la tabla 1, se detallan los ingredientes con sus correspondientes nutrientes; las diferentes formulaciones de las dietas experimentales, se presentan en la tabla 2 y la tabla 3 muestra el contenido nutricional calculado de las diferentes dietas utilizadas en el estudio.

Fase experimental: Cada acuario tenía una capacidad de 35L, dotados con filtro biológico y termostato, que mantenían controlada la temperatura del agua a $28 \pm$ $2^{\circ} \mathrm{C}$. Diariamente, se controlaba la temperatura y se realizaba un recambio de agua del $15 \%$; por semana, se lavaban los filtros y se efectuó un recambio del $80 \%$, de tal manera que permitiera reducir las concentraciones de amonio y de nitritos. Los tratamientos fueron asignados aleatoriamente. La fase experimental tuvo una duración de 32 días. Para medir el efecto de cada una de las dietas, los animales fueron pesados y medidos los días 0 y 33. El alimento suministrado, se calculó teniendo en cuenta el $15 \%$ de la biomasa sin realizar ningún; los animales eran alimentados tres veces al día (8:00 a.m., 12:00 m. y 16:00 p.m.), durante los 32 días de experimentación.

Análisis estadístico: Debido a la diferencia en el peso y la longitud estándar de los alevinos al comenzar el estudio, se utilizó un análisis de covarianza, para corregir el efecto inicial, todo esto bajo un diseño completamente al azar (Martínez \& Martínez, 1997), en un arreglo factorial 3 X2, para determinar los efectos de los tres niveles de PC (24, 28 y $32 \%$ ) con los dos niveles de harina de pescado (7,5 y $0 \%$ ) y sus interacciones en los parámetros: ganancia de peso expresada como porcentaje, tasa de crecimiento específico y ganancia en longitud estándar (distancia entre la parte anterior de la cabeza y comienzo de la aleta caudal). Para las interacciones $(P<0,05)$, se aplicó la prueba de Comparación de Medias, (Steel E Torrie, 1985). Todos los análisis estadísticos fueron realizados con la ayuda del programa SAS, (1989).

Tabla 1. Ingredientes utilizados para la formulación de las dietas de alevinos de bocachico con sus correspondientes nutrientes.

\section{NUTRIENTES EN LAS DIFERENTES MATERIA PRIMAS}

\begin{tabular}{|c|c|c|c|c|c|c|c|c|c|c|c|c|c|c|c|}
\hline Ingrediente & E.D. Kcal/kg & ${ }^{*} \mathrm{P} . \mathrm{C}$ & ${ }^{*} \mathrm{Ca}$ & ${ }^{*} \mathrm{P}$ & Lis & Met & Cis & Fen & Tir & Tre & Tri & Val & $\Omega 3$ & $\Omega 6$ & Na \\
\hline H Pescado & 4828 & 65,4 & 3,7 & 2,4 & 5 & 2 & 0,6 & 2,8 & 2 & 2,8 & 0,8 & 3,5 & & & 1,1 \\
\hline H Carne & 2930 & 55,6 & 8,3 & 4,1 & 2,9 & 0,7 & 0,6 & 1,7 & 1 & 1,6 & 0,3 & 2,5 & & & 1,2 \\
\hline T. Soya & 3010 & 48,5 & 0,3 & 0,6 & 3,1 & 0,7 & 0,8 & 2,4 & 2 & 1,9 & 0,7 & 2,6 & & & 0 \\
\hline Trigo & 2790 & 12,9 & 0 & 0,4 & 0,4 & 0,2 & 0,3 & 0,6 & 0 & 0,4 & 0,2 & 0,6 & & & 0 \\
\hline Maíz & 2200 & 8,5 & 0 & 0,3 & 0,3 & 0,2 & 0,2 & 0,5 & 0 & 0,4 & 0,1 & 0,4 & & & 0 \\
\hline Aceite Pesc. & 8000 & & & & & & & & & & & & 31 & 1,3 & \\
\hline Aceite Maíz & 7800 & & & & & & & & & & & & 0,7 & 58 & \\
\hline P. Vita. & & & & & & & & & & & & & & & \\
\hline P. Mine. & & & & & & & & & & & & & & & \\
\hline CO3Ca & & & & & & & & & & & & & & & \\
\hline
\end{tabular}

* Datos suministrados por la doctora Clara Salamanca, de Alimentos Concentrados Raza, los restantes fueron tomados de NRC, 1993. 
Tabla 2. Formulación de las dietas experimentales para alevinos de bocachico.

\begin{tabular}{|c|c|c|c|c|c|c|}
\hline & \multicolumn{3}{|c|}{ Sin Harina Pescado } & \multicolumn{3}{c|}{ Con Harina Pescado } \\
\hline Proteína \% & 24 & 28 & 32 & 24 & 28 & 32 \\
\hline INGREDIENTE & & & & & & \\
\hline Maíz & 33,3 & 30,17 & 22,87 & 40,51 & 35,91 & 28,84 \\
\hline Trigo & 17 & 12 & 10 & 17 & 12 & 10 \\
\hline T. Soya & 30,6 & 40,2 & 51,69 & 19,37 & 30 & 41,01 \\
\hline H Carne & 3,5 & 5 & 5 & 3,5 & 5 & 5 \\
\hline H Pescado & & & & 7,5 & 7,5 & 7,5 \\
\hline Aceite Maíz & 4 & 3,2 & 2,5 & 4 & 3,2 & 2,25 \\
\hline Aceite Pescado & 5,8 & 5,17 & 4,68 & 3,4 & 3,32 & 3,07 \\
\hline Carbonato Ca & 1,9 & 1,5 & 1,5 & 1,5 & 1 & 1 \\
\hline Fosfato de sodio & 0,93 & 0,48 & 0,26 & 0,33 & & \\
\hline P. Vita. . & 0,4 & 0,4 & 0,4 & 0,4 & 0,4 & 0,4 \\
\hline P. Mine. ${ }^{2}$ & 0,1 & 0,1 & 0,1 & 0,1 & 0,1 & 0,1 \\
\hline L-Lisina & 1,29 & 0,92 & 0,51 & 1,25 & 0,82 & 0,43 \\
\hline DL-Metionina & 0,43 & 0,33 & 0,21 & 0,38 & 0,26 & 0,14 \\
\hline L-Treonina & 0,56 & 0,4 & 0,22 & 0,55 & 0,35 & 0,19 \\
\hline L-Triptófano & 0,19 & 0,13 & 0,06 & 0,21 & 0,14 & 0,07 \\
\hline
\end{tabular}

1. Vitamina Rovimix peces Comp / kg. Vit. A 8 X 106 Ul; Vit. D $1.8 X 106$ Ul; Vit. E 66,66g Vit B1 6,66g; Vit B2 13,33g; Vit B6 6,66g; Ácido Pantoténico 33,33g; Biotina 533,3mg; Ácido Fólico 2,66g; Ácido Ascórbico 400,0g; Ácido Nicotínico 100,0g; Vit B12 20,0mg; Vit K 6,66g.

2. Premezcla minerales peces Roche S.A. Composición por 100g. Mn 10,0; Zn 16,0; Fe 4,0; Cu 1,0; I 0,5; Se 0,05; Co 0,01.

La ganancia de peso porcentual (GPP) y la tasa de crecimiento específico (TCE) fueron determinadas así:

$$
\begin{gathered}
G P P=\frac{(P F-P I)}{\mathrm{PI} * 100} \\
\mathrm{TCE}=\frac{(\text { In PF }- \text { In PI })}{\# \text { días }} * 100
\end{gathered}
$$

Donde:

$\mathrm{PF}=$ Peso Final $(\mathrm{g})$

PI $=$ Peso Inicial $(g)$

$\ln =$ Logaritmo neperiano

Ganancia en longitud estándar $(\mathrm{cm})=$ (long. están final - long. están inicial).

El modelo lineal utilizado fue: $Y_{i j}=\mu+\mathrm{HP}+\mathrm{PC}+(\mathrm{HP}$ $* P C)+\beta(P i)+\varepsilon i j$
Donde:

$\mu=$ Promedio poblacional

$\mathrm{HP}=$ Niveles de Harina de Pescado

$P C=$ Niveles de Proteína Cruda

$(\mathrm{HP} * \mathrm{PC})=$ Interacción

$\beta(\mathrm{Pi})=$ Efecto del peso inicial

$\varepsilon \mathrm{ij}=$ Error experimental

Teniendo como supuestos: los errores tienen una distribución normal e independiente con media cero y varianza $\sigma^{2}$.

Bajo las Hipótesis

Ho $=$ Todos los tratamientos son iguales

$\mathrm{Hi}=\mathrm{Al}$ menos uno es diferente 
Tabla 3. Contenido nutricional calculado de las dietas experimentales, para alevinos de bocachico.

\begin{tabular}{|c|c|c|c|c|c|c|}
\hline & \multicolumn{3}{|c|}{ Dietas sin Harina de Pescado } & \multicolumn{3}{c|}{ Dietas con Harina de Pescado } \\
\hline Proteína \% & $24 \%$ & $28 \%$ & $32 \%$ & $24 \%$ & $28 \%$ & $32 \%$ \\
\hline E.D. & 3105,3 & 3089,5 & 3093,9 & 3092,8 & 3114,4 & 3110,8 \\
\hline P.C. & 23,86 & 27,96 & 31,9 & 23,87 & 28,14 & 32,01 \\
\hline Ca & 1,15 & 1,13 & 1,16 & 1,24 & 1,19 & 1,21 \\
\hline P & 0,7 & 0,7 & 0,7 & 0,7 & 0,72 & 0,77 \\
\hline Arg & 1,5 & 1,86 & 2,24 & 1,41 & 1,8 & 2,16 \\
\hline His & 0,54 & 0,65 & 0,76 & 0,54 & 0,66 & 0,77 \\
\hline Isol & 0,92 & 1,11 & 1,32 & 0,94 & 1,15 & 1,35 \\
\hline Leu & 1,76 & 2,07 & 2,38 & 1,82 & 2,15 & 2,45 \\
\hline Lis & 2,2 & 2,23 & 2,23 & 2,22 & 2,23 & 2,23 \\
\hline Met + Cis & 1,14 & 1,14 & 1,15 & 1,13 & 1,14 & 1,14 \\
\hline Fen & 1,07 & 1,29 & 1,52 & 1,04 & 1,27 & 1,5 \\
\hline Tir & 0,76 & 0,91 & 1,08 & 0,75 & 0,92 & 1,08 \\
\hline Treo & 1,37 & 1,39 & 1,39 & 1,38 & 1,38 & 1,39 \\
\hline Trip & 0,47 & 0,47 & 0,47 & 0,47 & 0,47 & 0,47 \\
\hline Val & 1,12 & 1,35 & 1,6 & 1,12 & 1,38 & 1,62 \\
\hline S-3 & 1,84 & 1,64 & 1,48 & 1,1 & 1,07 & 0,98 \\
\hline$\Omega-6$ & 2,4 & 1,92 & 1,51 & 2,54 & 2,08 & 1,52 \\
\hline Na & 0,21 & 0,15 & 0,11 & 0,19 & 0,15 & 0,15 \\
\hline
\end{tabular}

E.D. = Energía Digestible Calculada Kcal/kg; Arg: Arginina; His: Histidina; Iso: Isoluecina; Lis: Lisina; Met: Metionina; Cis: Cistina; Fen: Fenilalanina; Tir: Tirosina; Treo: Treonina; Trip: Triptófano; Val: Valina.

\section{RESULTADOS Y DISCUSIÓN}

Con base en las medidas obtenidas del peso final, se calculó la ganancia de peso, expresada como porcentaje, y la tasa de crecimiento específico; en la tabla 4 se reportan los valores relacionados con los pesos de los alevinos. Al finalizar las cuatro semanas no se reportaron diferencias significativas $(P>0,05)$ entre los tratamientos. Al diseñar dietas para peces, se deben mejorar la eficiencia de la conversión proteica y buscar fuentes de proteína que minimicen el uso de la harina de pescado, es así como al evaluar los niveles de inclusión de la harina de pescado en las dietas $7,5 \%$ y $0 \%$, no se encontró diferencias significativa $(P>0,05)$, lo cual, coincide con lo reportado por Li et al. (2003), quienes no detectaron diferencias significativas en la ganancia de peso $(P>0,04)$, consumo de alimento $(P>0,12)$ o conversión alimenticia $(P>0,02)$ de los juveniles de catfish (Ictalurus punctatus), alimentados con dietas que contenían $0 \%, 6 \%$ y $12 \%$ de harina de pescado; así mismo, Li et al. (2006), utilizaron dietas con $4 \%$ y $8 \%$ de harina de pescado y no reportan diferencias en el consumo de alimento y ganancia de peso en juveniles de catfish. Gonzáles et al. (2007) concluyen que los alevinos de tilapia nilótica, se adaptan fácilmente a las dietas libres de harina de pescado y no muestran diferencias significativas en la ganancia de peso y tasa de crecimiento especifico, con dietas enriquecidas con la harina de pescado. Así mismo, Civera et al. (2006) reportan la ausencia diferencias estadísticas para las 
variables peso y longitud en juveniles de tilapia al realizar remplazos parcial y total de la harina de pescado por harina de cerdo. En contraste, Mohsen E Lovell (1990) encontraron que suministrando dietas con 5 a $10 \%$ de harina de pescado a channel catfish se presentan diferencias significativas en ganancia de peso y eficiencia alimentaria comparadas con aquellas carentes de ésta, aún cuando sean suplementadas con aceite de pescado. Li et al. (2006) concluyen que la mejor ganancia de peso en juveniles de catfish, se obtuvo cuando las dietas que contenían un $4 \%$ de harina de pescado; sin embargo, cuando se incremento a un $8 \%$, no se presenta mejor desempeño.

Tabla 4. Pesos iníciales, pesos finales, ganancia de peso expresada como porcentaje y tasa de crecimiento específico de los alevinos de bocachico, sometidos a diferentes dietas.

\begin{tabular}{|c|c|c|c|c|c|c|}
\cline { 2 - 7 } \multicolumn{1}{c|}{} & $24 \%$ Sin HP & $24 \%$ Con HP & $28 \%$ Sin HP & $28 \%$ Con HP & $32 \%$ Sin HP & $32 \%$ Sin HP \\
\hline Peso Inicial (g) & $1,39 \pm 0,01$ & $4,2 \pm 0,05$ & $1,43 \pm 0,04$ & $1,42 \pm 0,04$ & $4,19 \pm 0,11$ & $4,11 \pm 0,06$ \\
\hline Peso Final (g) & $2,58 \pm 0,21$ & $6,21 \pm 0,15$ & $2,9 \pm 0,28$ & $3,05 \pm 0,21$ & $5,78 \pm 0,23$ & $6,67 \pm 0,37$ \\
\hline G Peso \%* & $85,71 \pm 15,77$ & $47,96 \pm 5,08$ & $103,14 \pm 19,24$ & $115,11 \pm 19,11$ & $38,14 \pm 7,63$ & $62,13 \pm 6,79$ \\
\hline TCE $^{* *}$ & $1,87 \pm 0,26$ & $1,19 \pm 0,11$ & $2,14 \pm 0,29$ & $2,31 \pm 0,22$ & $0,98 \pm 0,17$ & $1,46 \pm 0,12$ \\
\hline
\end{tabular}

Nota: Son promedios con su desviación estándar.

* Ganancia de peso porcentual

** Tasa de crecimiento específico

En la tabla 4, se observa que, con relación a la ganancia de peso expresado, como porcentaje, no se presentaron diferencias significativas $(\mathrm{P}>0,05)$, lo que coincide con lo referido por Pezzato et al. (2000), en alevinos de piauçu (Leporinus macrocephalus), alimentados con dietas de 24,28 y $32 \%$ de proteína, con un $4 \%$ de inclusión de harina de pescado. Los resultados obtenidos en el presente experimento son inferiores a los reportados por González \& Wills (2005).

Shiau E Lan (1996), trabajando con ocho dietas (0, 8, 16, 24, 32, 40, 48, 56\% PC), para la alimentación de juveniles de mero (Epinephelus malabaricus), reportan ganancias porcentuales de peso para los niveles de proteína de 24 y $32 \%$, de 172,1 y $247,5 \%$, respectivamente, teniendo como base de la proteína a la harina de pescado; el período experimental duro ocho semanas. Es de anotar que las ganancias de peso fueron aumentando en la medida que se incrementó la proteína, hasta alcanzar un nivel de 48\%. Así mismo, Massamitu et al. (2005) consideran que en tilapias no hay efecto significativo entre dietas que contienen 25,5; $27 ; 28,5$ y $30 \%$ de proteína digestíble
En la variable tasa de crecimiento específico, no se observaron diferencias estadísticas $(P>0,05)$ en los efectos principales ni en la interacción (Tabla 4). Estos resultados contrastan lo reportado por $\mathrm{Li} \&$ Robinson (1999) quienes reportan diferencias $(P<0,05)$ para esta variable entre los juveniles de catfish alimentados con dietas con tres niveles de proteína (24, 28 Y 32\% PC).

En la tabla 5, se pueden distinguir los valores relacionados con la longitud estándar. La longitud estándar final al igual que la ganancia en longitud estándar, no presentaron diferencias estadísticas $(P>0,05)$ entre los diferentes tratamientos.

Referente a la suplementación con los aminoácidos sintéticos vale la pena resaltar que no se observaron efectos en la ganancia de peso expresada como porcentaje o en la longitud estándar, lo cual, coincide con lo reportado por Li \& Robinson (1998), quienes suministraron dietas enriquecidas con lisina y metionina a juveniles de Catfish y no lograron incrementar la ganancia de peso ni mejorar la composición corporal, en juveniles de tilapia. De la misma manera, Zarate $\mathcal{E}$ 
Tabla 5. Longitud estándar inicial, final y ganancia en longitud estándar de los alevinos de bocachico

\begin{tabular}{|c|c|c|c|c|c|c|}
\cline { 2 - 6 } \multicolumn{1}{c|}{} & $24 \%$ & $24 \%$ & $28 \%$ & $28 \%$ & $32 \%$ & $32 \%$ \\
\hline $\begin{array}{c}\text { Long Está } \\
\text { Inicial (cm) }\end{array}$ & $3,93 \pm 0,07$ & $5,39 \pm 0,21$ & $4,09 \pm 0,21$ & $4,00 \pm 0,13$ & $5,40 \pm 0,15$ & $5,43 \pm 0,16$ \\
\hline $\begin{array}{c}\text { Long Está } \\
\text { Final (cm) }\end{array}$ & $4,74 \pm 0,12$ & $6,18 \pm 0,22$ & $4,85 \pm 0,15$ & $5,01 \pm 0,17$ & $6,01 \pm 0,32$ & $6,41 \pm 0,28$ \\
\hline $\begin{array}{c}\text { Gan. Long } \\
\text { Están (cm) }\end{array}$ & $0,81 \pm 0,17$ & $0,79 \pm 0,10$ & $0,76 \pm 0,26$ & $1,01 \pm 0,12$ & $0,61 \pm 0,21$ & $0,98 \pm 0,17$ \\
\hline
\end{tabular}

Nota: Son promedios con su desviación estándar, ns: no significativo.

Ganancia en longitud estándar $(\mathrm{cm})$ = (longitud estándar final - longitud estándar inicial).

Lovell (1997), concluyen que la suplementación de las dietas con lisina sintética no mejora los parámetros productivos de los juveniles de catfish. Saavedra et al. (2009) manifiestan que la suplementación de las dietas con triptófano y lisina no mejoran el crecimiento de las larvas, debido, probablemente, a la inhibición del apetito generado por una baja palatabilidad. Por otro lado, Furuya et al. (2004) consideran que en juveniles de tilapia es importante la suplementación de aminoácidos sintéticos, cuando las dietas sean carentes de harina de pescado.

Bajo las condiciones reportadas, se puede concluir que los alevinos de bocachico aceptan las dietas con bajos niveles de harina de pescado o, mejor aún, sin la inclusión de ésta, lo cual, es un paso importante para mitigar, de alguna manera, el exagerado uso de esta materia prima en las dietas de esta especie. Esto demuestra, que estos peces, que pertenecen a la baja escala trófica acuícola, pueden mostrar el mismo desempeño de los animales alimentados con dietas enriquecidas con harina de pescado, en esta etapa fisiológica.

Adicionalmente, el haber obtenido resultados similares con dietas que tenían un contenido proteico más bajo favorecería al productor, en el sentido de poder disminuir el costo de la dieta. Por lo anterior, se puede asegurar que los alevinos de bocachico pueden presentar un desempeño adecuado cuando se suministran dietas carentes de harina de pescado y con una proteína del $24 \%$ siempre y cuando se garantice un nivel adecuado de aminoácidos.
AGRADECIMIENTOS: Los autores quieren expresar su más sincero agradecimiento a la doctora Nhora Martínez, docente de Bioestadística de la Facultad de Medicina Veterinaria y de Zootecnia de la Universidad Nacional, por su asesoría en la parte estadística del trabajo. Conflictos de intereses: El manuscrito fue preparado y revisado con la participación de todos los autores, quienes declaramos que no existe ningún conflicto de intereses que ponga en riesgo la validez de los resultados presentados.

\section{BIBLIOGRAFÍA}

1. ABDEL-TAWWAB, M., AHMAD, M.H., 2009. Effect of dietary protein regime during the growing period on growth performance, feed utilization and whole-body chemical composition of Nile Tilapia, Oreochromis niloticus (L.). Aquaculture Res. 40:1532-1537.

2. ABIMORAD, E.G.; FAVERO, G.C.; CASTELLANI, D.; GARCIA, F.; CARNEIRO, D.J. 2009. Dietary supplementation of lysine and/or methionine on performance, nitrogen retention and excretion in pacu Piaractus mesopotamicus reared in cages. Aquaculture. 295(3,4):266-270.

3. BARRETO, C.; MOSQUIERA, B. 2001. Boletín Estadístico Pesquero Colombiano. INPA, Bogotá. p.142

4. CIVERA, R.C; GOYTORTUA, B.E.; ROCHA, M.S.; ASTORGA, R. D.; ÁLVAREZ, C.A.; AVALOS H,N. 2006. Evaluación de la sustitución parcial y total de 
harina de sardina con harina de cerdo en alimentos para juveniles de la tilapia del nilo Oreochromis niloticus: efecto sobre la supervivencia, el crecimiento y la utilización del alimento. Centro de investigaciones biológicas del noroeste, s. c. Universidad Juárez Autónoma de Tabasco. Informe técnico final. 24p.

\section{FOOD AND AGRICULTURE ORGANIZATION OF} THE UNITED NATIONS (FAO). 2006. The States of world fisheries and aquaculture. Roma, Italy. p.180.

6. FURUYA, W.M; PEZZATO, L.E.; BARROS, M.M; PEZZATO, A.C.; FURUYA, V.R.B.; MIRANDA.E. 2004. Use of ideal protein concept for precision formulation of amino acid levels in fish-meal-free diets for juvenile Nile tilapia (Oreochromis niloticus L.) Aquaculture Res. 35:1110-1116.

7. GABER, M.M. 2006. The effects of plant-protein-based diets supplemented with yucca on growth, digestibility, and chemical composition of Nile Tilapia (Oreochromis niloticus, L) Fingerlings. J. World Aquaculture Society. 37(1):74-82.

8. GONZÁLES, J.M.; HUTSON, A.H.; ROSINSKI, M.E.; BROW, P.B. 2007. Evaluation of fish meal-free diets for first feeding Nile Tilapia, Oreochromis niloticus. J. Applied Aquaculture. 19(3):89-99.

9. GONZÁLEZ U., R.A.; WILLS F., G.A. 2005. Relación proteína/energía digestible para alevinos de bocachico (Prochilodus magdalenae), Steinachner, 1878. Rev. U.D.C.A Act. E Div. Cient. (Colombia). 8(2):111-118.

10. KIKUCHI, K.; FURUTA, T. 2009. Use of defatted soybean meal and blue mussel meat as substitute for fish meal in the diet of Tiger Puffer, Takifugu rubripes. J. World Aquaculture Society. 40(4):472482.

11. LI, M.H.; PETERSON, C.B.; JANES, C.L.; ROBINSON, E.H. 2006. Comparison of diets containing varíous fish meal levels on growth performance, body composition, and insulin-like growth factor-I of juvenile channel catfish Ictalurus punctatus of different straits. Aquaculture. 253:628-635.
12. LI, M.H.; WISE, D.J.; MANNING, B.B.; ROBINSON, E.H. 2003. Effect of dietary total protein and animal protein on growth and feed efficiency of channel catfish Ictalurus punctatus and their response to Edwardsiella ictaluri challenge. J. World Aquaculture Society. 34:223-228.

13. LI, M.H.; ROBINSON, E.H. 1999. Effect of reducing dietary digestible energy to protein ratio on weight gain body fat of juvenile channel catfish Ictalurus punctatus. J. World Aquaculture Society. 30(1):123-127.

14. LI, M.H.; ROBINSON, E.H. 1998. Effects of supplemental lysine and methionine in low protein diets on weight gain and body composition of young channel catfish (Ictalurus punctatus). Aquaculture. 163:297-307.

15. LOVELL, T. 1989. Nutrition and feeding of fish. Auburn University. Library of Congress Cataloging in Publication Data. p.260.

16. MARTÍNEZ, B.R.; MARTÍNEZ, R.N. 1997. Diseño de Experimentos. Análisis de datos estándar y no estándar. $1^{\mathrm{a}} \mathrm{ed}$. Fondo Nacional Universitarío. (Colombia). p.479.

17. MASSAMITU, W.; BOTARO, D.; GOMES DE MACEDO, R.M.; GOMES DOS SANTOS, V.; SILVA, L.C.R.; CASTRO, T.; BARRIVIERA, F.V.R.; PINSETA, S.P.J. 2005. Aplicação do conceito de proteína ideal para redução dos níveis de proteína em dietas para tilápia-do-nilo (Oreochromis niloticus). R. Bras. Zootec. 34(5):1433-1441.

18. MILES, C. 1974. Los peces del río Magdalena. Ministerio de la Economía Nacional. Secretaría de Piscicultura, Pesca y Caza. Editorial el Gráfico. Bogotá, Colombia. p.242.

19. MOHSEN, A.A.; LOVELL, R.T. 1990. Partial substitution of soybean meal with animal protein sources in diets for channel catfish. Aquaculture. 90:303-311.

20. MOJICA, J.I.; ÁLVAREZ-LEÓN, R. 2002. Prochilodus magdalenae. En : Mojica, J.I.; Castellanos, C.; Usma, S.; Álvarez, R. (Eds). Libro Rojo de peces 
dulceacuícolas de Colombia. La serie Libros Rojos de Especies Amenazadas de Colombia. Instituto de Ciencias Naturales Universidad Nacional de Colombia, Ministerio del Medio Ambiente, Bogotá, Colombia. p.285.

21. NATIONAL RESEARCH COUNCIL NRC. Nutrient Requirements of Fish. 1993. Committee on Animal Nutrition. Board on Agriculture. National Academy Press. Washington. 1993. p.116.

22. PERES, H.; OLIVA-TELES, A. 2005. The effect of dietary protein replacement by crystalline amino acido growth and nitrogen utilization of turbot Scophthalmus maximus juveniles. Aquaculture. 250:755-764.

23. PEZZATO, L.E.; BARROS, H.M.; PEZZATO, A.C.; MIRANDA, E.C.; QUINTERO, P.L.G.; FURUYA, W.M. 2000. Relación energía: proteína en la nutrición de alevinos de piaucu (Leporinus macrocephalus). Rev. Med. Vet. y Zoot. (Colombia). 47:2-6.

24. SAAVEDRA, M.; BARR,Y.; POUSSÃO-FERREIRA, P.; HELLAND, S.; YUFERA, M.; DINIS, M.T.; CONCEICÃO, L.E.C. 2009. Supplementation of tryptophan and lysine in Diplodus sargus larval diet: effects on growth and skeletal deformities. Aquaculture Res. 40:1191-1201.

25. SALHI, M.; BESSONART, M.; CHEDIAK, G.; BELLAGAMBA, M.; CARNEVIA, D. 2004. Growth, feed utilization and body composition of black catfish, Rhamdia quelen, fry fed diets containing different protein and energy levels. Aquaculture. 231:435-444.
26. STATISTICAL ANALYSIS SYSTEM (SAS). 1989. Sas/Stat User's guide, version 6, 4th ed. vol I SAS Institute, Cary, NC.

27. SHIAU, S.Y.; LAN, C.W. 1996. Optimum dietary protein level and protein to energy ratio for growth of grouper (Epinephelus malabaricus). Aquaculture. 145:259-266.

28. STEEL, R.; TORRIE, J. 1985. Bioestadística: Principios y procedimientos. Traducida de la $1^{\mathrm{a}}$ edición inglesa por Ricardo Martínez (2a ed.). Bogotá, Colombia. McGraw-Hill. p.622.

29. TWIBELL, R.G.; GRIFFIN, M.E.; MARTIN, B.; PRICE, J.; BROWN, P.B. 2003. Predicting dietary essential amino acid requirements for hybrid striped bass. Aquac. Nutr. 9:373-381.

30. WATANABE, T.; AOKI, H.; WATANABE, K.; MAITA, M.; YAMAGATA, Y.; SATOH, S. 2001. Quality evaluation of different types of non-fish meal diets for yellowtail. J. Fish Sci. 67:461-469.

31. YAMAMOTO, T.; SHIMA, T.; FURUITA, H.; SHIRAISHI, M.; SÁNCHEZ, V.; TABATA, M. 2000. Self-selection of diets with different amino acid profiles by rainbow trout Oncorhynchus mykiss. Aquaculture. 187:375-386.

32. ZARATE, D.D.; LOVELL, R.T. 1997. Bioavailability of free vs. protein-bound lysine in practical diets for young channel catfish (Ictalurus punctatus). Aquaculture. 159:87-100.

Recibido: Marzo 17 de 2009

Aceptado: Noviembre 18 de 2009 